Received: 2019.06.23 Accepted: 2019.12.16 Available online: $\quad 2020.02 .17$ Published: 2020.03.31

\section{Treatment of a Gastrointestinal Stromal Tumor (GIST) Adherent to the Spleen and the Tail of the Pancreas: A Case Report}

e-ISSN 1941-5923

C) Am J Case Rep, 2020; 21: e918278

DOI: 10.12659/AJCR.918278
Authors' Contribution: Study Design A Data Collection B Statistical Analysis C Data Interpretation D Manuscript Preparation E Literature Search F Funds Collection G

\author{
ABCDEFG 1 Christoforos S. Kosmidis \\ ABCDEFG 1 Vyron Alexandrou \\ ABCDEFG 1 Georgios D. Koimtzis \\ $A B C 1$ Stylianos Mantalovas \\ AE 1 Nikolaos C. Varsamis \\ AE 1 Charilaos Koulouris \\ AE 2 David Taraboulous \\ AE 3 Ariadne Leptopoulou \\ AE 3 Eleni Georgakoudi \\ FG 3 Christina D. Sevva \\ AE 1 Konstantinos Sapalidis \\ B 4 Sophia Lypiridou \\ B 4 Georgia Karayannopoulou \\ AE 1 Isaac I. Kesisoglou
}

$13^{\text {rd }}$ Surgical Department, University Hospital of Thessaloniki AHEPA, Thessaloniki, Greece

2 Department of Gastroenterology, Bioclinic SA, Thessaloniki, Greece 3 University Hospital of Thessaloniki AHEPA, Aristotle University of Thessaloniki, Thessaloniki, Greece

4 Department of Pathology, University Hospital of Thessaloniki AHEPA, Thessaloniki, Greece
Corresponding Author: Conflict of interest:
Vyron Alexandrou, e-mail: vyrwnal@hotmail.com None declared
Patient:

Final Diagnosis:

Symptoms:

Medication:

Clinical Procedure:

Specialty:

Objective:

Background:

Case Report:

Conclusions:

\section{MeSH Keywords:}

Full-text PDF:

\title{
Male, 62-year-old
}

Gastrointestinal stromal tumor (GIST) adherent to the spleen and the tail of the pancreas Melena

En bloc sphenoid gastrectomy • splenectomy • caudal pancreatectomy

Surgery

\section{Rare disease}

Gastrointestinal stromal tumors (GISTs) are the most common mesenchymal gastrointestinal tumors (GIT). Usually, they appear in patients ages 55-65 years, with no apparent difference between males and females. Their annual incidence is about 11-14 per $10^{6}$. They generally do not present with any prominent symptoms, appearing with the atypical symptoms of abdominal pain, weight loss, early satiety, and occasionally bleeding. Adequate surgical treatment involves sphenoid resection of the tumor within clear margins. If adjacent organs are involved, en bloc resection is the procedure of choice.

A 62-year-old male patient presented to the Emergency Department complaining of melena for 1 week. He underwent gastroscopy, colonoscopy and abdominal computed tomography scan, which revealed a large, exophytic, lobular mass $(12.6 \times 9.7 \times 12 \mathrm{~cm})$ of the greater curvature of the stomach. The patient underwent en bloc sphenoid gastrectomy, splenectomy, and caudal pancreatectomy. The histopathologic examination revealed findings compatible with a gastrointestinal stromal tumor located at the stomach, with low-grade malignancy (G1) and T4NO according to TNM classification. He was discharged from the hospital on the $7^{\text {th }}$ postoperative day.

GISTs are uncommon tumors of the gastrointestinal system that usually do not invade neighboring organs or develop distant metastases; therefore, local resection is usually the treatment of choice. However, in cases of large GISTs that are adherent to neighboring organs, en bloc resection and resection of adjacent organs may be inevitable.

Gastrointestinal Stromal Tumors • Pancreas • Spleen • Stomach Neoplasms

https://www.amjcaserep.com/abstract/index/idArt/918278

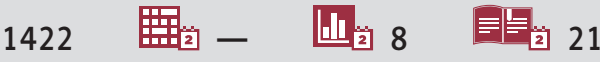




\section{Background}

Gastrointestinal stromal tumors (GISTs) are a type of mesenchymal tumor that appear in the gastrointestinal tract, originating from the Cajal interstitial cells [1]. In the USA, the incidence of GISTs is around 11-14 per $10^{6}$, while in Europe recent estimates put the incidence at around the same values $\left(1: 10^{5}\right)$. The mean age at diagnosis is $55-60$ years, with no apparent difference between males and females [2,3]. It is estimated that more than half of GISTs (50-60\%) are in the stomach, $30 \%$ are in the jejunum or ileum, $5 \%$ are in the duodenum, $5 \%$ are in the rectum, and less than $1 \%$ are in the esophagus [4]. Approximately 1 in 3 GISTs are asymptomatic and are detected incidentally during screening imaging studies or abdominal surgical procedures. Symptomatic GISTs appear with gastrointestinal bleeding (varying from insidious chronic bleeding to life-threatening acute bleeding), usually along with gastric discomfort mimicking the symptoms of an ulcer. Few GISTs manifest as other abdominal emergencies, such as intestinal obstruction, or with rupture resulting in hemoperitoneum [4]. The preferred treatment for GISTs is total surgical resection of the tumor. In cases of large tumors and invasion of neighboring organs, en bloc resection of the tumor together with the neighboring organs might be needed [5].

We present the case of a 62-year-old man who was diagnosed with GIST. Due to its adhesion to the pancreas and the spleen, he underwent en bloc sphenoid gastrectomy, splenectomy, and caudal pancreatectomy.

\section{Case report}

A 62-year-old man presented to the Emergency Department complaining of melena for 1 week accompanied by feeling of early satiety and weight loss of approximately $10 \mathrm{~kg}$ in the span of 2 months, with no related pain, abdominal discomfort, or palpable abdominal masses. He was initially admitted to the Gastroenterology Department, where he underwent laboratory and imaging studies. All the laboratory results, including the tumor markers, were within normal values. Imaging studies included gastroscopy, colonoscopy, and abdominal computed tomography (CT) scan. The colonoscopy did not reveal any abnormal lesions, while the gastroscopy showed a submucosal mass with mucosal ulceration in the greater curvature of the stomach (Figure 1), and the CT scan (Figure 2) revealed a large lobular mass $(12.6 \times 9.7 \times 12 \mathrm{~cm})$ originating from the greater curvature of the stomach, with adhesion to the neighboring organs (spleen and pancreas). He was transferred to our Surgical Department, where he underwent exploratory laparotomy, which revealed an exophytic mass of the stomach, in agreement with the findings of the CT scan (Figures 3,4). Therefore, en bloc sphenoid gastrectomy, splenectomy, and

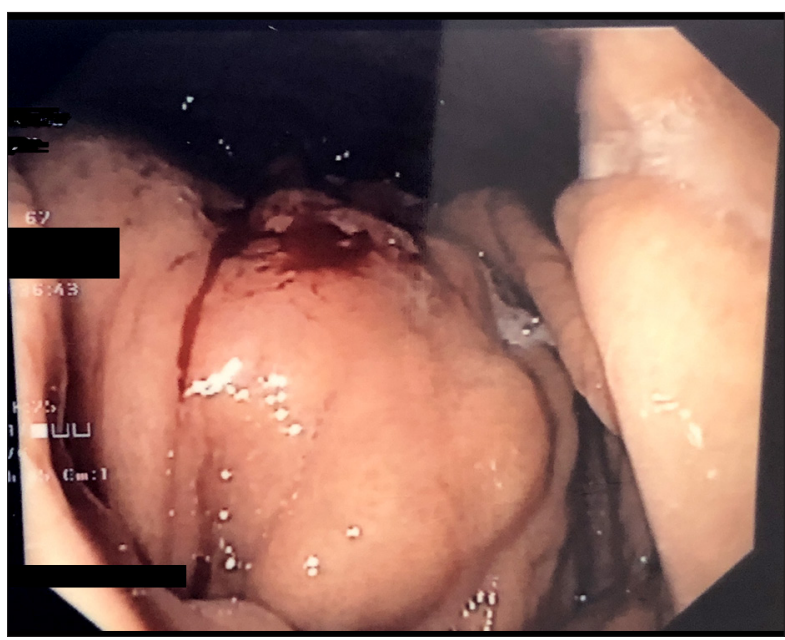

Figure 1. Gastroscopy image of the submucosal mass and ulceration on the greater curvature of the stomach.

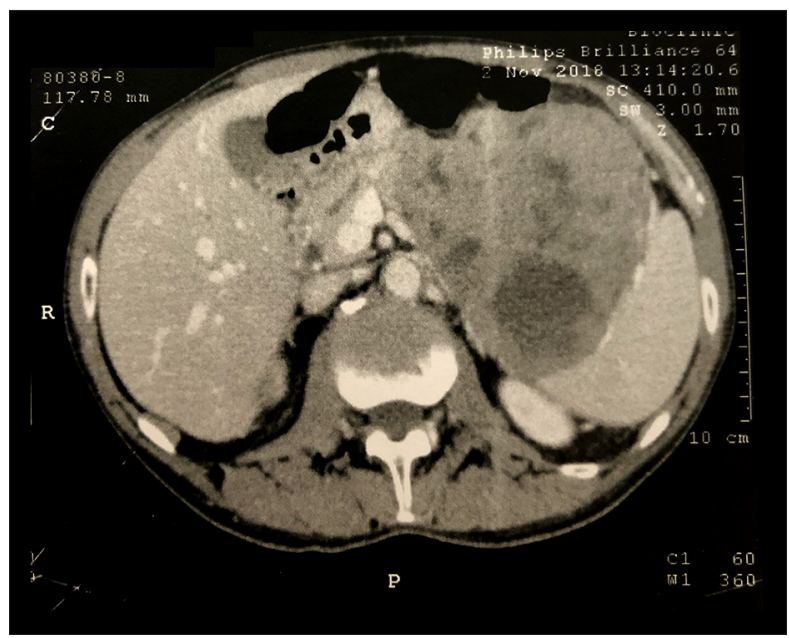

Figure 2. CT scan image showing a large lobular mass originating from the greater curvature of the stomach.

caudal pancreatectomy were performed (Figure 5). A drainage tube was placed in the left subdiaphragmatic space at the site of the splenectomy. The patient recovered safely in the operating room and was transferred back to the floor.

The patient's postoperative course was uneventful. The nasogastric tube was removed on the $4^{\text {th }}$ postoperative day and the drainage tube was removed 2 days later. Oral intake of liquids began on the $4^{\text {th }}$ postoperative day and he was discharged on the $7^{\text {th }}$ postoperative day.

The histopathologic examination of the specimen revealed histologic and immunochemical findings compatible with low-grade (G1) gastric GIST, staged pT4pN0 according to the TNM staging system. Specifically, the immunochemical control showed strong positivity for C-KIT, DOG1, and caldesmon neoplastic cells, and weaker positivity for SMA and negative for 


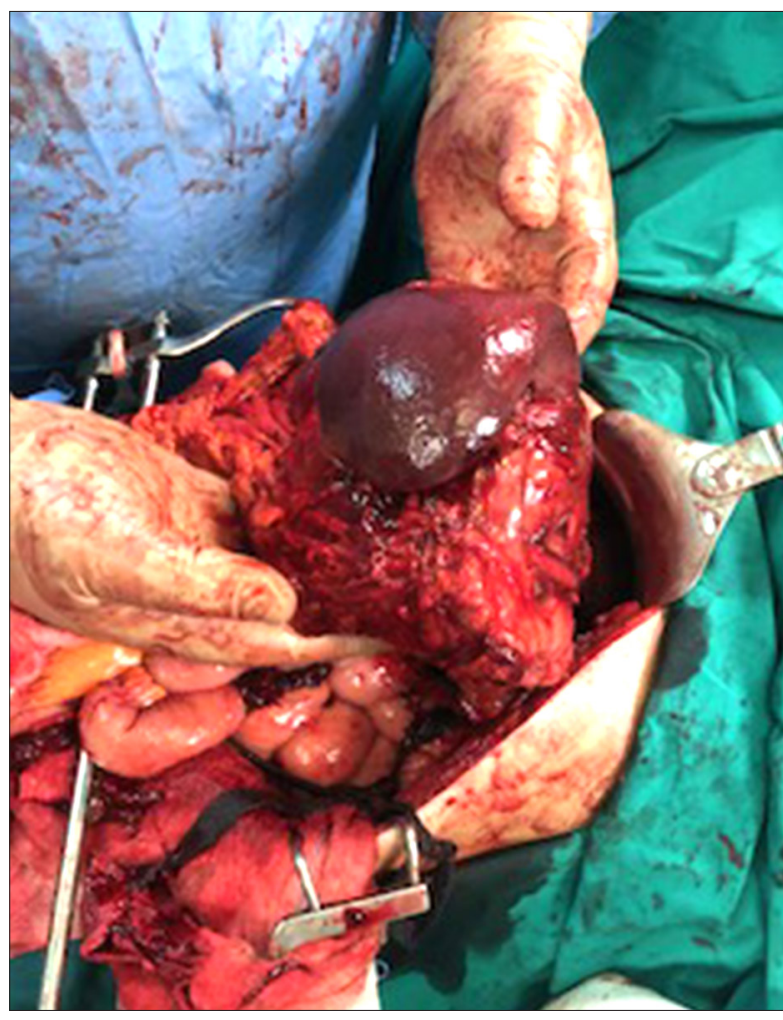

Figure 3. Intraoperative image showing adhesion of the tumor to the spleen.

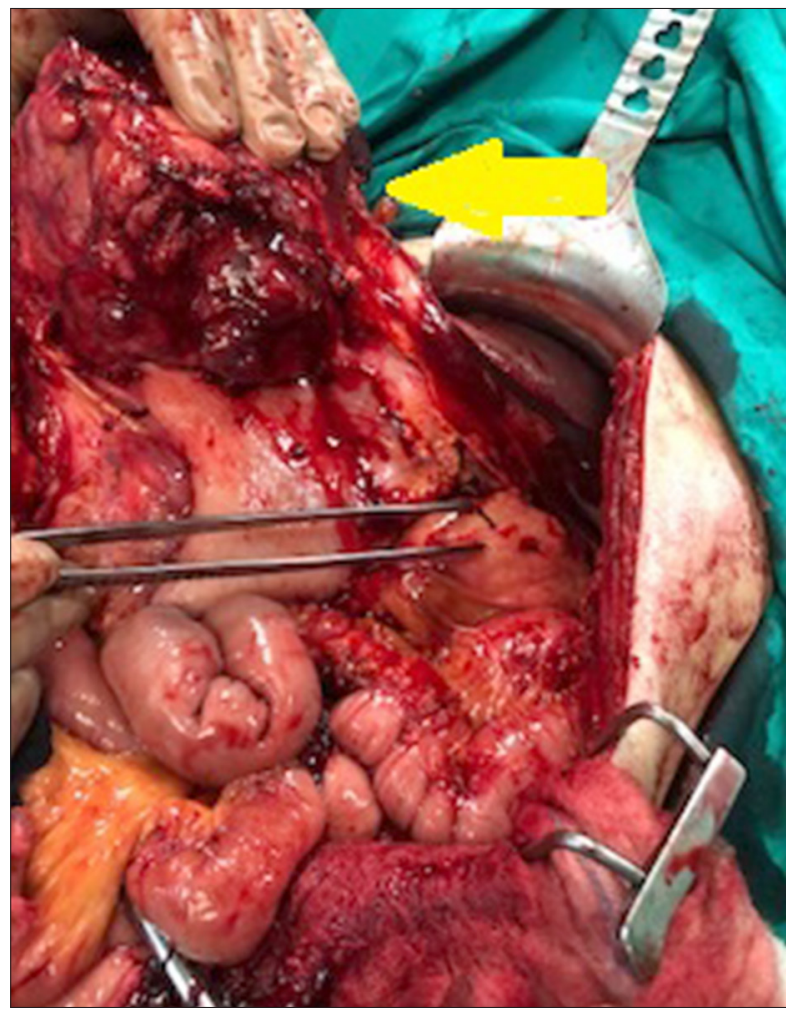

Figure 4. Intraoperative image showing the tumor (yellow arrow) and its adhesion to the pancreas (forceps).

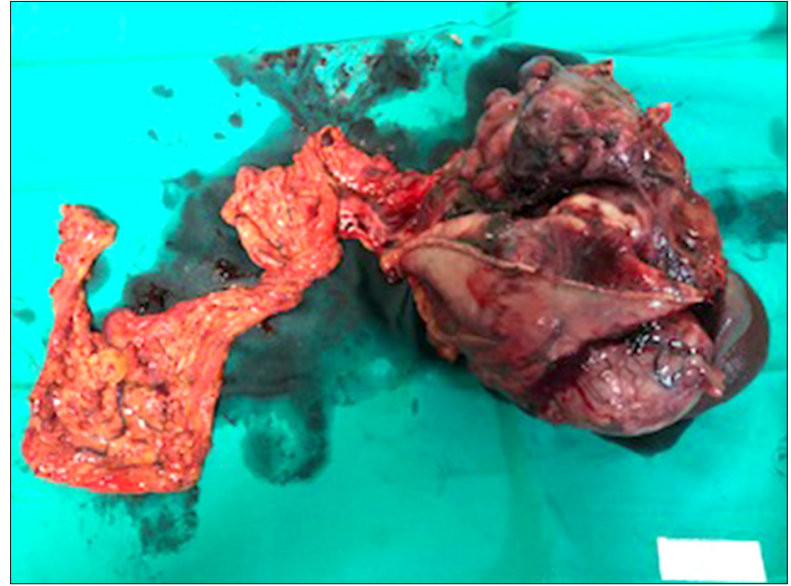

Figure 5. The specimen after being excised.

desmin, CD34 antigen, keratin 8/18, and S100 protein. The mitotic index KI67/MIB1 was positive up to $2 \%$ in the majority of the neoplastic cells, but there were a few sites where it reached $15 \%$. The margins of the stomach were free of neoplastic disease, and the spleen and the pancreas did not show evidence of invasion from the tumor. Gross pathological examination of the specimen revealed a $21-\mathrm{cm}$ nodular mass, tan-white on sectioning, confined to the gastric submucosa, without invasion of the gastric mucosa. Histological examination of this lesion showed a tumor composed of spindle or polygonal cells, with eosinophilic cytoplasm, poorly-defined cell membranes, and round or elongated nuclei forming palisades, with occasional perinuclear vacuolization. The neoplastic cells were arranged in intersecting bundles and occasionally in whorls. A few necrotic spots were observed. The mitotic rate was low (1 mitosis per 50 high-power fields), and there was no evidence of tumor cells in surgical margins (R0 excision). Neither the pancreatic nor the splenic parenchyma were invaded by the neoplasm. Immunohistochemically, neoplastic cells were diffuse and strongly stained for C-KIT (CD117), DOG1, and caldesmon, weakly stained for smooth muscle actin (SMA), and not stained for desmin and CD34. The Ki67-labeling index ranged from $2 \%$ to $15 \%$, with a median of $3.3 \%$ (Figures $6-8$ ).

After an oncologic consultation, the patient was started on $400 \mathrm{mg}$ of imatinib per day and he remains disease-free 6 months postoperatively.

\section{Discussion}

GISTs are mesenchymal tumors originating from the intestinal cells of Cajal and can be found in every part of the gastrointestinal tract [1]. Usually, they are localized tumors $<5 \mathrm{~cm}$ in diameter, with extremely low occurrence of lymph node metastases [6-8]. The most common sites of distant metastases for gastric GISTs are the peritoneal cavity and liver. Few cases 


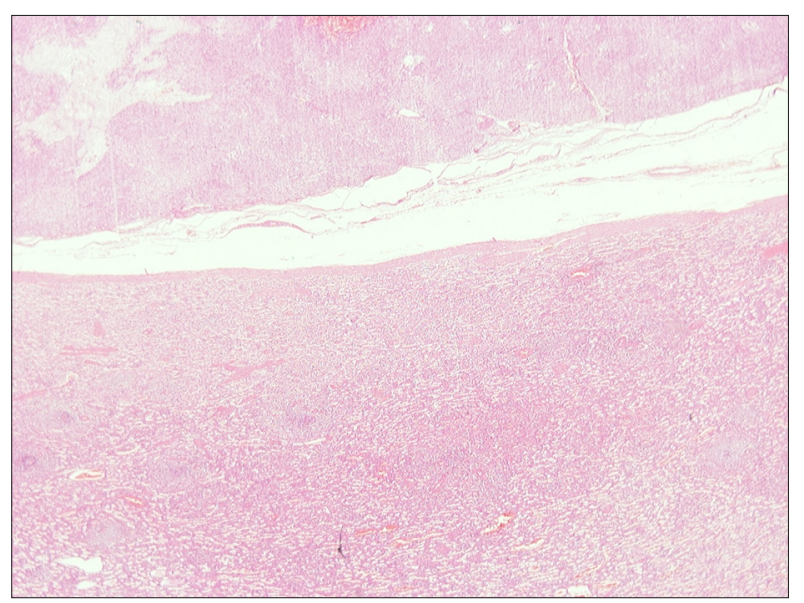

Figure 6. $(\mathrm{HE} \times 40)$ Pancreatic parenchyma without neoplastic involvement.

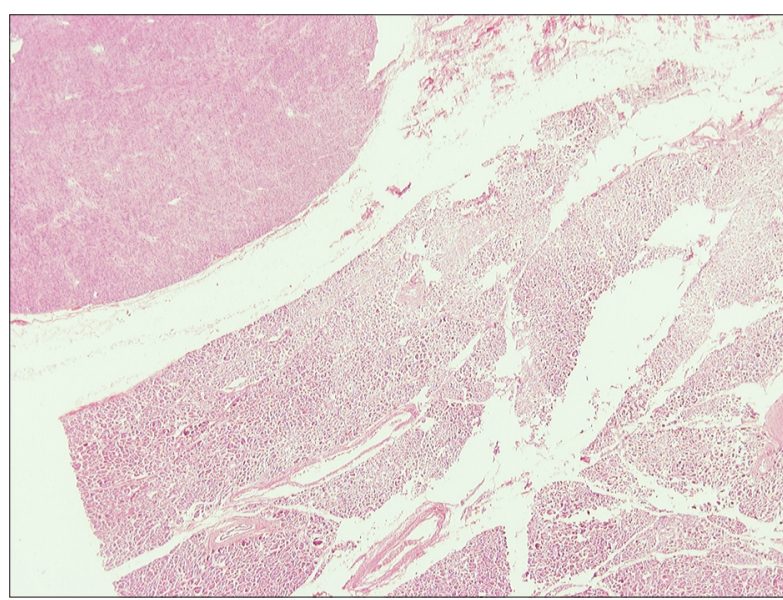

Figure 7. $(\mathrm{HE} \times 20)$ Neoplastic tissue without gastric-mucosal involvement.

of distant metastases to bones have been observed, predominantly on the axial skeleton, usually the spine. Metastases to the surrounding cutaneous and peripheral soft tissues are very rare; in contrast to other malignant sarcomas, lung metastases almost never occur [4]. Consequently, the standard treatment for gastric GISTs is wedge or segmental resection of the tumor. The main goal is to avoid tumor rupture and to achieve clear margins of excision (R0) with the fewest possible post-surgical comorbidities [2,9].

In a retrospective study by Lanping Zhu et al., endoscopic resection of 250 GISTs less than $2 \mathrm{~cm}$ in diameter was reported. Apart from 67 cases of intraoperative bleeding managed endoscopically with surgical clips and nylon bands, no other major complications were reported during their 8.5 -year postoperative surveillance period. During this period, $80.2 \%$ of patients reported improvement of symptoms and no recurrence or metastasis was found [10]. The same tumor size of $2 \mathrm{~cm}$ is reported as a criterion for endoscopic resection in a systematic

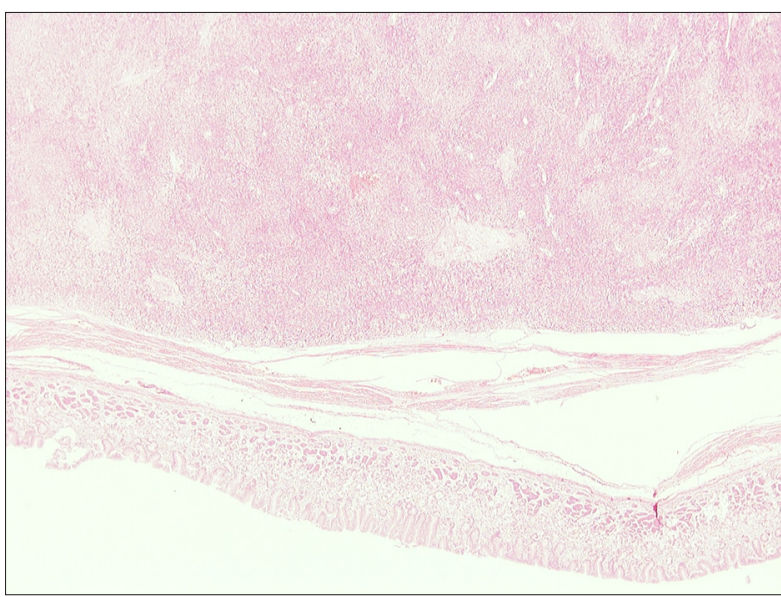

Figure 8. $(\mathrm{HE} \times 20)$ Splenic parenchyma is not involved by neoplastic tissue.

review by Qiang Zhang et al., who gathered data from 11 different studies investigating endoscopic resection of GISTs, reporting a recurrence rate up to $5.8 \%$ after a follow-up period of 32 months [11]. In contrast, a study by Changji Yu in 2017 proposed endoscopic full-thickness resection for the treatment of GISTs up to $5 \mathrm{~cm}$ in diameter [12]. Regarding laparoscopic resection of GISTs, a study published in 2017 suggested that the upper limit for laparoscopic excision of GISTs is $5 \mathrm{~cm}$ in diameter, with some researchers reporting laparoscopic excisions in tumors $5-10 \mathrm{~cm}$ in diameter $[13,14]$.

However, in cases in which either the tumor size or its location renders a RO surgical excision not feasible or when it there is increased risk of post-surgical morbidity, neo-adjuvant treatment with imatinib, attempting to shrink the tumor and facilitate its excision, has been recommended $[8,15]$. Nonetheless, in our case, due to acute gastrointestinal bleeding presenting with melena, emergency surgery was elected over neo-adjuvant therapy in accordance with the recent literature [16]. In cases in which there is adherence or infiltration of the surrounding organs by the tumor, en bloc excision of the tumor with all the involved surrounding structures is recommended [9]. Therefore, in our case, following the recommended surgical guidelines, we performed en bloc sphenoid gastrectomy, splenectomy, and caudal pancreatectomy.

Generally, infiltrating GISTs are rarely encountered. According to a report by Magdy et al., among 49 gastric GISTs, only 2 required partial gastrectomy, splenectomy, and distal pancreatectomy, while Weili Yang et al. reported 2 more cases that needed multivisceral en bloc resection, one with partial colectomy and another with distal pancreatectomy and splenectomy $[17,18]$. Another case report, published in 2016, reported resection of a GIST en bloc with the spleen and a full-thickness cuff of the diaphragm [19]. Finally, 2 more case reports, one by Kondo et al. and one by Namikawa et al., reported cases similar to ours, 
with gastric GISTs that required en bloc splenectomy and caudal pancreatectomy. However, in these 2 cases, the operations were performed after long-term therapy with imatinib [20,21].

\section{Conclusions}

GISTs are uncommon tumors of the gastrointestinal system that are usually asymptomatic and are often found incidentally.

\section{References:}

1. Starczewska Amelio JM, Cid Ruzafa J, Desai K et al: Prevalence of gastrointestinal stromal tumour (GIST) in the United Kingdom at different therapeutic lines: An epidemiologic model. BMC Cancer, 2014; 14: 364

2. Rammohan A, Sathyanesan J, Rajendran K et al: A gist of gastrointestinal stromal tumors: A review. World J Gastrointest Oncol, 2013; 5(6): 102-12

3. Casali P G, Abecassis N, Bauer S et al: ESMO Guidelines Committee and EURACAN; Gastrointestinal stromal tumours: ESMO-EURACAN Clinical Practice Guidelines for diagnosis, treatment and follow-up. Ann Oncol, 2018; 28(4): 68-78

4. Miettinen M, Lasota J: Gastrointestinal stromal tumors. Gastroenterol Clin North Am, 2013; 42(2): 399-415

5. Poveda A, del Muro XG, López-Guerrero JA et al: GEIS 2013 guidelines for gastrointestinal sarcomas (GIST). Cancer Chemother Pharmacol, 2014; 74(5): 883-98

6. Nishida T, Blay JY, Hirota $\mathrm{S}$ et al: The standard diagnosis, treatment, and follow-up of gastrointestinal stromal tumors based on guidelines. Gastric Cancer, 2015; 19(1): 3-14

7. Akahoshi K, Oya M, Koga T, Shiratsuchi Y: Current clinical management of gastrointestinal stromal tumor. World J Gastroenterol, 2018; 24(26): 2806-17

8. Sanchez-Hidalgo JM, Duran-Martinez M, Molero-Payan R et al: Gastrointestinal stromal tumors: A multidisciplinary challenge. World J Gastroenterol, 2018; 24(18): 1925-41

9. Koo DH, Ryu MH, Kim KM et al: Asian Consensus Guidelines for the diagnosis and management of gastrointestinal stromal tumor. Cancer Res Treat, 2016; 48(4): 1155-66

10. Zhu L, Khan S, Hui Y et al: Treatment recommendations for small gastric gastrointestinal stromal tumors: Positive endoscopic resection. Scand J Gastroenterol, 2019; 54(3): 297-302
They are usually small and thus can be removed endoscopically or laparoscopically, utilizing the advantages of minimally invasive surgery. However, in cases of large GISTs that are adhering to neighboring organs, en bloc resection of adjacent organs such as the spleen or pancreas may be required.

\section{Conflict of interest}

None.

11. Zhang Q, Gao LQ, Han ZL et al: Effectiveness and safety of endoscopic resection for gastric GISTs: A systematic review, Minim Invasive Ther Allied Technol, 2018; 27(3): 127-37

12. Yu C, Liao G, Fan C et al: Long-term outcomes of endoscopic resection of gastric GISTs. Surg Endosc, 2017; 31(11): 4799-804

13. Lee CM, Park S: Laparoscopic techniques and strategies for gastrointestinal GISTs. J Vis Surg, 2017; 3: 62

14. Chi J-L, Xu M, Zhang M-R, Li Y, Zhou Z-G: Laparoscopic versus open resection for gastric gastrointestinal stromal tumors (GISTs): A size-locationmatched case-control study. World J Surg, 2017; 41(9): 2345-52

15. Iwatsuki M, Harada K, Iwagami S et al: Neoadjuvant and adjuvant therapy for gastrointestinal stromal tumors. Ann Gastroenterol Surg, 2018; 3(1) 43-49

16. Liu Q, Kong F, Zhou J et al: Management of hemorrhage in gastrointestinal stromal tumors: A review. Cancer Manag Res, 2018; 10: 735-43

17. Sorour MA, Kassem MI, Ghazal Ael-H et al: Gastrointestinal stromal tumors (GIST)-related emergencies. Int J Surg, 2014; 12(4): 269-80

18. Yang W, Yu J, Gao Y et al: Preoperative imatinib facilitates complete resection of locally advanced primary GIST by a less invasive procedure. Med Oncol, 2014; 31(9): 133

19. Palanivelu C, Rangarajan M, Annapoorni S: Segmental gastric resection with splenectomy for a malignant gastrointestinal stromal tumour of the stomach. Hellenic J Surg, 2016; 88: 119

20. Kondo J, Chijimatsu H, Kijima T et al: [A case of gastric GIST with pathological complete response achieved by long-term chemotherapy with imatinib mesylate.] Gan To Kagaku Ryoho, 2018; 45(3): 515-17 [in Japanese]

21. Namikawa T, Munekage E, Munekage $M$ et al: Synchronous large gastrointestinal stromal tumor and adenocarcinoma in the stomach treated with imatinib mesylate followed by total gastrectomy. Anticancer Res, 2016; 36(4): 1855-59 